\title{
A RANDOMISED ALGORITHM FOR CHECKING THE NORMALITY OF CRYPTOGRAPHIC BOOLEAN FUNCTIONS
}

\author{
An Braeken, Christopher Wolf, and Bart Preneel \\ K.U.Leuven, ESAT-COSIC \\ Kasteelpark Arenberg 10 \\ B-3001 Leuven-Heverlee, Belgium \\ http://www.esat.kuleuven.ac.be/cosic/ \\ \{An.Braeken, Christopher.Wolf, Bart.Preneel\}@esat.kuleuven.ac.be
}

\begin{abstract}
A Boolean function is called normal if it is constant on flats of certain dimensions. This property is relevant for the construction and analysis of cryptosystems. This paper presents an asymmetric Monte Carlo algorithm to determine whether a given Boolean function is normal. Our algorithm is far faster than the best known (deterministic) algorithm of Daum et al. In a first phase, it checks for flats of low dimension whether the given Boolean function is constant on them and combines such flats to flats of higher dimension in a second phase. This way, the algorithm is much faster than exhaustive search. Moreover, the algorithm benefits from randomising the first phase. In addition, by evaluating several flats implicitly in parallel, the time-complexity of the algorithm decreases further.
\end{abstract}

Keywords: Normality, Boolean Functions, Asymmetric Monte Carlo, Cryptography

\section{Introduction}

\subsection{Motivation}

Boolean functions and maps play a central role in cryptology. They are basic building blocks of bit-oriented block and stream ciphers. In order to construct secure cryptographic ciphers, i.e., ciphers which resist all known attacks, it is important to study the structure and behaviour of Boolean functions.

Normality of a Boolean function is the property which determines if the function is constant on a flat of dimension $\lceil n / 2\rceil$. This concept was introduced by Dob94, in order to construct highly nonlinear balanced Boolean functions. Later, this property was used to distinguish different classes of bent functions. As the first bent function which is non-normal occurs for dimension 14 (Can03), we need a highly optimised algorithm for determining the normality of Boolean functions. This is non-trivial as 
the total number of flats increases exponentially for increasing dimension $\boldsymbol{n}$ (MWS91). Table 1 lists the number of flats of dimension $[n / 2\rceil$; this clearly shows that even for moderate dimensions ( $n \geq 13 \ldots 15$ ) establishing normality by exhaustive search is infeasible.

Table 1. The number of flats of dimension $\left\lceil\frac{n}{2}\right\rceil$ to test for different dimensions $n$

\begin{tabular}{|r||r|r|r|r|r|r|r|r|r|r|r|r|r|}
\hline$n$ & 8 & 9 & 10 & 11 & 12 & 13 & 14 & 15 & 16 & 17 & 18 & 19 & 20 \\
\hline $\log _{2}$ (\# flats) & 22 & 26 & 32 & 37 & 44 & 50 & 58 & 65 & 74 & 82 & 92 & 101 & 112 \\
\hline
\end{tabular}

\subsection{Related Work}

The first attempt for determining the normality of a Boolean function, better than exhaustive search, is due to DDL03. The main idea of their algorithm is to search exhaustively all flats of small dimension on which the function is constant and then to combine these to flats of higher dimension.

\subsection{Achievement}

In our algorithm, we replace the exhaustive search through all flats of small dimension by a random search. This has several advantages over the algorithm of Daum et al. First, we do not need a unique representation of flats which means less conditions to test and therefore a lower time complexity. Second, the number of repetitions needed to determine with high probability that a function is non-normal, is far smaller than an exhaustive search on all flats of small dimension (cf Sect. 4.2). Our algorithm is of the asymmetric Monte Carlo type and may output "non-normal" with probability $2^{-c}$ for a normal function and some confidence level $c \in \mathbb{N}$. The output "normal" is always correct. This asymmetric Monte Carlo algorithm has a far smaller running time than the deterministic algorithm of DDL03 — even with a reasonable error-probability ( $c=80$ in our case).

\subsection{Outline}

This paper is organised as follows. In Sect. 2, we introduce the basic definitions together with a description of the main ideas in our algorithm. Sect. 3 presents more details and explains several optimisations for our algorithm. In Sect. 4, we give a detailed complexity analysis of the algorithm and compare the total time complexity of our algorithm with the time complexity of the previous algorithm from DDL03. This paper concludes with Sect. 5 .

\section{Background}

In this section we present some definitions and a simplified algorithm to test the normality of a Boolean function. 


\subsection{Definitions}

Before we can describe our algorithm, we need to define several objects. We start with vectors and vector spaces and finish with some definitions concerning Boolean functions.

Let a vector $\bar{u} \in \mathbb{F}_{2}^{n}$ be represented by the $n$-tuple $\left(u_{n-1}, \ldots, u_{0}\right)$ with the coefficients $\boldsymbol{u}_{\boldsymbol{i}} \in \mathbb{F}_{\mathbf{2}}$ from the field with 2 elements. Let $\overline{\boldsymbol{u}_{1}}, \ldots, \overline{\boldsymbol{u}_{\boldsymbol{k}}} \in \mathbb{F}_{\mathbf{2}}^{\mathbf{n}}$ be $\boldsymbol{k}$ linearly independent vectors. Then they form the base of the subspace

$$
\langle U\rangle:=\left\langle\overline{u_{1}}, \ldots, \overline{u_{k}}\right\rangle:=\left\{\alpha_{1} \overline{u_{1}} \oplus \ldots \oplus \alpha_{k} \overline{u_{k}} \mid \alpha_{i} \in \mathbb{F}_{2}\right\} .
$$

Here, the dimension of $\langle U\rangle$ is $k$. For a given vector $\bar{a} \in \mathbb{F}_{2}^{n}$, we represent the coset of this subspace by $U_{\bar{a}}:=\bar{a} \oplus\langle U\rangle$. Throughout this paper, we call the coset $U_{\bar{a}}$ a flat. The vector $\bar{a}$ of the flat $U_{\bar{a}}$ is called the offset of this flat. In addition, two flats are said to be parallel if they are cosets of the same subspace $\langle U\rangle$, i.e., all flats of the form $U_{\bar{a}}, \bar{a} \in \mathbb{F}_{2}^{n}$ are parallel flats by this definition. Finally, we denote the set of all flats of dimension $\boldsymbol{s}$ by Flat $_{\boldsymbol{s}}$, i.e.,

$$
\text { Flat }_{s}:=\left\{U_{\bar{a}} \mid \bar{a} \in \mathbb{F}_{2}^{n},\langle U\rangle \subseteq \mathbb{F}_{2}^{n}, \operatorname{dim}\langle U\rangle=s\right\} .
$$

We now move on to Boolean functions. A Boolean function $f$ is a mapping from $\mathbb{F}_{2}^{n}$ into $\mathbb{F}_{2}$. The property of normality for a Boolean function $f$ is defined as follows:

DEFINITION 1 A Boolean function $f: \mathbb{F}_{\mathbf{2}}^{n} \rightarrow \mathbb{F}_{\mathbf{2}}$ is called normal if there exists a flat $W_{\bar{a}} \subset \mathbb{F}_{2}^{n}$ of dimension $\lceil n / 2\rceil$ such that $f$ is constant on $W_{\bar{a}}$, i.e., $\forall \bar{w} \in W_{\bar{a}}$ : $f(\bar{w})=c$ for some fixed $c \in\{0,1\}$. We call the flat $\boldsymbol{W}_{\overline{\mathbf{a}}}$ a witness for the normality of the function $f$.

As we see from Definition 1, the property of normality is related to the question of the highest dimension of the flats on which the function $f$ is constant. As a consequence, it is natural to generalise the previous definition by the introduction of $k$ normality (Dub01; Car01):

DEFINITION 2 For a natural number $k: 1 \leq k \leq n$, a Boolean function $f: \mathbb{F}_{2}^{n} \rightarrow$ $\mathbb{F}_{2}$, is said to be "k-normal" if there exists a flat $V_{\mathfrak{a}} \in$ Flat $_{k}$ such that $\boldsymbol{f}$ is constant on $V_{\bar{a}}$, i.e., $\forall \bar{v} \in V_{\bar{a}}: f(\bar{v})=c$ for some fixed $c \in\{0,1\}$. We call the flat $V_{\bar{a}} a$ " $k$-witness" for the normality of the function $f$.

Remark: It is clear that a constant function $f(\bar{x})=c, \forall \bar{x} \in \mathbb{F}_{2}^{n}, c \in \mathbb{F}_{2}$ is $n$-normal. An affine function $f(\bar{x})=\bar{a} \cdot \bar{x} \oplus b, \forall \bar{x}, \bar{a} \in \mathbb{F}_{2}^{n}, b \in \mathbb{F}_{2}$ is $(n-1)$-normal, because it is normal on the flats $\{\bar{x}: \bar{a} \cdot \bar{x} \oplus b=0\}$ and $\{\bar{x}: \bar{a} \cdot \bar{x} \oplus b=1\}$ of dimension $n-1$.

\subsection{A Simple Algorithm}

The previous section shows that it is important for the definition of normality and $k$-normality, i.e., for a given dimension $e:=k$ ( $k$-normality) or $e:=\lceil n / 2\rceil$ (ordinary normality), to find a witness $W_{\bar{a}} \in$ Flat $_{\boldsymbol{e}}$. To ease the understanding of the algorithm of Sect. 4, we start with a highly non-optimised version of it (cf Fig. 1). Both algorithms are based on the observation made by DDL03, that a Boolean function which is constant on a flat $W_{\bar{a}}$ is also constant on all flats contained in $W_{\bar{a}}$, i.e., $f_{\mid W_{\bar{a}}}=c$ for 
EXAMPLE 3 Consider some parallel flats of the following subspace of dimension 2 which is defined by $\langle U\rangle:=\langle(0,0,1),(0,1,0)\rangle \subseteq \mathbb{F}_{\mathbf{2}}^{\mathbf{3}}$.

$$
\begin{aligned}
(1,0,0) \oplus\langle(0,0,1),(0,1,0)\rangle & =(1,1,0) \oplus\langle(0,0,1),(0,1,0)\rangle \\
& =(1,1,1) \oplus\langle(0,0,1),(0,1,0)\rangle \\
& =(1,0,1) \oplus\langle(0,0,1),(0,1,0)\rangle
\end{aligned}
$$

As a consequence, the parallel flats can be divided into equivalence classes. Therefore, we use the complement of a subspace $\langle U\rangle$, i.e., the subspace $\langle\bar{U}\rangle$ which satisfies

$$
\langle\bar{U}\rangle \oplus\langle U\rangle=\mathbb{F}_{2}^{n} \text { and }\langle\bar{U}\rangle \cap\langle U\rangle=\{0\} .
$$

This allows us to determine the representatives of the equivalence classes of the parallel flats, namely the flats $U_{\bar{a}}$, for $\bar{a} \in\langle\bar{U}\rangle$. Because the dimension of $\langle\bar{U}\rangle$ is equal to $n-\boldsymbol{s}$, there are in total $2^{n-\boldsymbol{s}}$ different parallel flats. To compute the complement $\langle\bar{U}\rangle$ of a given subspace $\langle U\rangle$ efficiently, we make use of the Permuted Gauss Basis (PGB) of a subspace. To define the PGB, we need to introduce the concept of left-most-one of a vector first.

DEFINITION 4 For a given vector $\bar{u}=\left(u_{n-1}, \ldots, u_{0}\right)$, we define the left-most-one as the position of the left-most one in its representation:

$$
\nu(\bar{u}):=\min \left\{i \in\{-1, \ldots, n-1\} \mid u_{j}=0 \text { for } i<j \leq n\right\} .
$$

DEFINITION 5 The vectors $\overline{u_{1}}, \ldots, \overline{u_{k}}$ form a PGB basis iff

$$
\nu\left(\overline{u_{i}}\right) \neq \nu\left(\overline{u_{j}}\right), \quad 0 \leq i<j<n .
$$

Remark: The name Permuted Gauss Basis is motivated as follows. Thinking about the base vectors $\overline{u_{1}}, \ldots, \overline{u_{k}}$ as a matrix, we would perform Gaussian elimination on it, without swapping rows. The result would not be a triangular structure but a row permutation.

For a subspace $\langle U\rangle$, we denote the set of the different left-most-ones of its elements

$$
\Upsilon(<U>):=\{\nu(\bar{u}) \mid \bar{u} \in<U>\backslash\{0\}\}
$$

The complement $\langle\bar{U}\rangle$ of a subspace $\langle U\rangle$ where $\langle U\rangle$ is in PGB can be computed as follows:

$$
<\bar{U}>=\left\{\bar{a} \in \mathbb{F}_{2}^{n} \mid a_{i}=0, \text { where } i \in \Upsilon(<U>)\right\}
$$

\subsection{Random Points instead of Random Bases}

Instead of selecting a random flat with a PGB, we choose $(s+1)$ points at random. This is cheaper than selecting a vector space at random which satisfies the PGBcriterion. In addition, we only need to transfer a set of $(s+1)$ points into a PGB if the function $f$ is constant on the corresponding flat. As this only happens with probability $2^{-2^{4}+1}$, we obtain very low costs on average. For $\boldsymbol{s}$ points, we can compute 
Figure 2. Algorithm for computing the PGB of a set of points

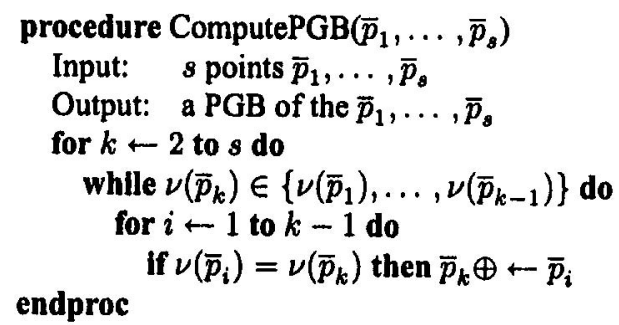

the PGB by the iterative algorithm from Fig. 2. The point $\bar{p}_{0}$ is the offset of the flat $\bar{p}_{0} \oplus\left\langle\bar{p}_{1}, \ldots, \bar{p}_{s}\right\rangle$ and has to be reduced as outlined in the previous section.

Finally, we have to check whether the $(s+1)$ points form a flat of dimension $\boldsymbol{s}$. The contrary happens only with very small probability:

$$
\left(2^{n}\right)\left(2^{n}-1\right) \cdots\left(2^{n}-2^{s}\right) / 2^{n \cdot(s+1)} \text {. }
$$

Using the following strategy, we can reduce the running time of the algorithm further: instead of picking $(s+1)$ points at random and evaluate explicitly if they form a flat of dimension $\boldsymbol{s}$ on which the function $f$ is constant, we do this implicitly in parallel:

- Pick $(2 s+1)$ points at random

- Evaluate $f$ on these points

- if exactly $(s+1)$ points evaluate to 1 (resp. to 0 ), check if the corresponding flat yields the constant 1 (resp. 0 ) on the function $f$.

This implicit evaluation strategy exploits different observations. First, we assume that we can form a total of \#flats $:=\left(\begin{array}{c}2 s+1 \\ s+1\end{array}\right)$ independent flats of dimension $s$ using a set of $(2 s+1)$ points. This way, we can decrease the number of repetitions by this factor. In addition, we observe that a set of $(2 s+1)$ points will yield at most one flat of dimension $\boldsymbol{s}$ on which the function $f$ is constant, if $(s+1)$ points in the set evaluate to 1 (resp. 0 ) on the function $f$. However, the probability for this event is rather high, namely $\operatorname{Pr}($ only one flat $):=\frac{2\left(\begin{array}{c}2 a+1 \\ \theta+1\end{array}\right)}{2^{2} s+1}$.

But there is a price to pay for this strategy: we always need to perform $(2 s+1)$ evaluations of the function $f$ and also the same number of random calls.

Remark: It is natural to generalise this idea to other values than $(2 s+1)$. However, in this case we do not obtain such a good trade-off between the factor \#flats and the workload to check the corresponding flats. The choice $(2 s+1)$ is optimal for the given problem.

\subsection{Combining}

In the original algorithm, we searched for all parallel flats and started a recursion on each of them. This is obviously superfluous as we will find the same witness several 
times this way. As we know from the previous section, we will obtain at least $\mathbf{2}^{\boldsymbol{e}-\boldsymbol{s}}$ parallel flats $U_{\bar{b}_{i}}$ on which the function is constant. Here, $\boldsymbol{e}$ denotes the end-dimension and $\boldsymbol{s}$ the start-dimension.

To avoid this costly computation, we use a different strategy, based on DDL03: instead of recursively searching for all parallel flats of higher dimension, we combine flats of low dimension to obtain flats of higher dimension. This is based on the following observation:

$$
\left(\bar{b}_{i} \oplus\langle U\rangle\right) \cup\left(\bar{b}_{j} \oplus\langle U\rangle\right)=\bar{b}_{i} \oplus\left\langle U, \bar{b}_{i} \oplus \bar{b}_{j}\right\rangle .
$$

Hence, we only need to consider pairs $\left(\bar{b}_{i}, \bar{b}_{j}\right) \in\langle\bar{U}\rangle \times\langle\bar{U}\rangle$ which lead to the same sum and then combine them recursively until we obtain a flat ofdimension $\boldsymbol{e}$. To do this efficiently, we introduce $2^{n}$ lists (depending on a vector $\bar{v} \in \mathbb{F}_{2}^{n}$ ) which hold an offset for each possible sum, i.e., $\operatorname{Append}\left(L^{\bar{b}_{i} \oplus \overline{b_{i}}}, \overline{b_{i}}\right)$. In the following section, we develop a branching condition for the combine method, which allows to decrease its running time even further.

\subsection{Branching}

Let the function $f$ take a constant value $c \in\{0,1\}$ on the flat $U_{\bar{a}}$ of dimension $d$. Denote with $P\left(U_{\bar{a}}\right)$ the set of all flats parallel to $U_{\bar{a}}$ on which the function yields the same constant. The following branching condition defined by the cardinality of the set $P\left(U_{\bar{a}}\right)$ has been observed by DDL03. We are able to improve their result by giving a shorter proof.

THEOREM 6 If $\left|P\left(U_{\bar{a}}\right)\right|<\mathbf{2}^{\mathbf{e}-\boldsymbol{d}}$, we can terminate the current branch of the combinemethod in $\langle U\rangle$ without violating its correctness.

Proof: Let $\boldsymbol{W}_{\bar{b}}$ be a witness and $U_{\bar{a}} \subset W_{\bar{b}}$ its subwitness. Now, there exist exactly $(e-d)$ linearly independent vectors $\overline{w_{1}}, \ldots, \overline{w_{e-d}} \in<W>$ with $\overline{w_{1}}, \ldots, \overline{w_{e-d}} \notin<$ $U>$ and consequently $\overline{w_{1}}, \ldots, \overline{w_{e-d}} \in\langle\bar{U}\rangle$. These vectors exist due to dimension reasons as $\operatorname{dim} W_{\bar{b}}=e$ and $\operatorname{dim} U_{\bar{a}}=d$. Therefore, for any subwitness $U_{\bar{a}} \subset W_{\bar{b}}$ exist $2^{\boldsymbol{e}-\boldsymbol{d}}$ parallel subwitnesses. This implies that $\left|P\left(U_{\bar{a}}\right)\right| \geq 2^{\boldsymbol{e}-\boldsymbol{d}}$. As a consequence, we can stop at any step in the algorithm if this condition is violated because we will not be able to extend the flat $U_{\bar{a}}$ to a witness of dimension $\boldsymbol{e}$.

\section{The Improved Algorithm}

Using the ideas from the previous section, we obtain the algorithm of Fig. 3. The method SearchForParallelFlats can be found in Fig. 4 and the optimised version of the combine-method is presented in Fig. 5. In the following sections, we analyse this optimised algorithm.

\subsection{Complexity Analysis}

We start the analysis of the algorithm with determining the number $\boldsymbol{r}$ of repetitions. Then we analyse the complexity of the main loop from Fig. 3, the complexity of the SearchForParallelFlats from Fig. 4 and the complexity of the Combine-procedure from Fig. 5 in different steps. 
Figure 3. Main loup for the optimised algorithm

Input: function $f$, start dimension $s$, end dimension $e$, repetitions $r$ Output: one witness if the function is $e$-normal

for $i \leftarrow 1$ to $r$ do

$S_{0} \leftarrow\{\}, S_{1} \leftarrow\{\}$

for $i \leftarrow 1$ to $2 s+1$ do

$\bar{p} \in \mathbb{R}_{R} \mathbb{F}_{2}^{n}$

$c \leftarrow f(\bar{p})$

$S_{c} \cup \leftarrow\{\bar{p}\}$

endfor

if $\left(\left(\left|S_{0}\right| \neq s+1\right)\right.$ and $\left.\left(\left|S_{1}\right| \neq s+1\right)\right)$ then continue $c \leftarrow\left|S_{1}\right|-s$

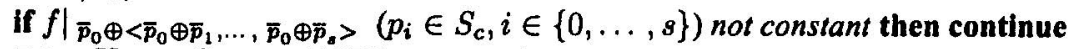

$\bar{a} \oplus\langle U\rangle \leftarrow \operatorname{ComputePGB}\left(\overline{p_{0}}, \ldots, \overline{p_{s}}\right)$

if $\operatorname{dim}\langle U\rangle \neq s$ then continue

SearchForParallelFlats $(<U>)$

endfor

Figure 4. SearchForParallelFlats for the optimised algorithm

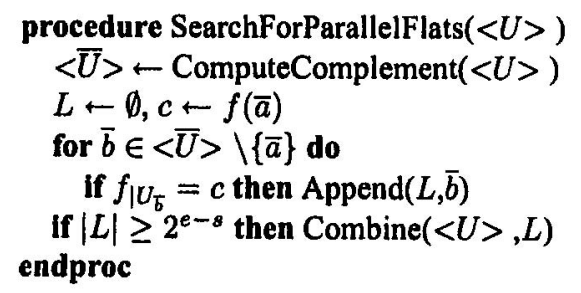

\section{Number of Repetitions.}

For determining the number of repetitions, we need the following lemma from MWS91, concerning the number of subspaces and flats of a certain dimension in a vector space.

LEMMA 7 The number of subspaces of dimension $s$ in a vector space of dimension $n$ is given by

$$
N S(n, s):=\prod_{i=0}^{s-1} \frac{2^{n-i}-1}{2^{s-i}-1}
$$

The number of flats of dimension $\boldsymbol{s}$ in a vector space of dimension $n$ is given by

$$
N F(n, s):=2^{n-s} \prod_{i=0}^{s-1} \frac{2^{n-i}-1}{2^{s-i}-1}=2^{n-s} N S(n, s) .
$$

Before determining a bound on $r$, we first introduce the term complaisant flat. 
Figure 5. Combine-method for the optimised algorithm

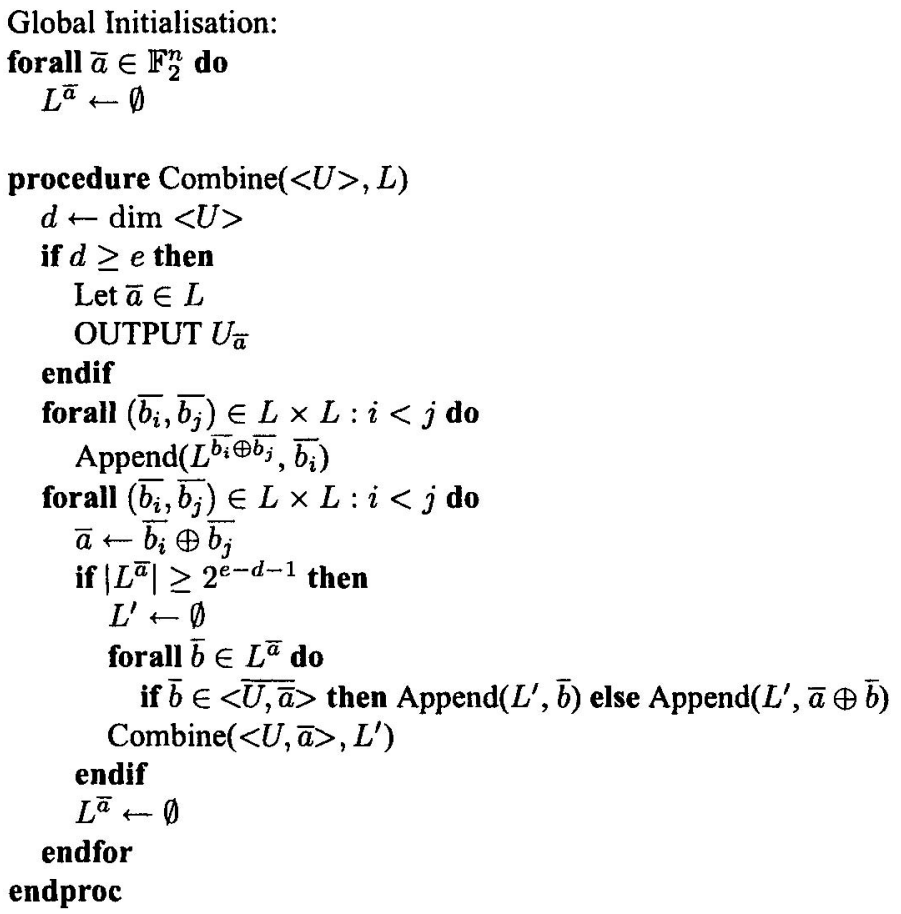

DEFINITION $8 \boldsymbol{A}$ flat $\boldsymbol{U}_{\overline{\mathbf{a}}}$ is called complaisant if the function is constant on the flat, the flat is parallel to a sub-witness, but the flat is not contained in any witness.

THEOREM 9 When choosing $(s+1)$ points $\bar{p}_{0}, \ldots, \bar{p}_{\mathbf{s}} \in \mathbb{F}_{\mathbf{2}}^{\mathbf{n}}$ at random, the probability $\boldsymbol{P F}(n, \boldsymbol{s}, e)$ that the flat $\boldsymbol{U}_{\overline{\boldsymbol{a}}}$ formed by these $(\boldsymbol{s}+\mathbf{1})$ points pass the first step in the algorithm is equal to

$$
\operatorname{PF}(n, s, e)=\operatorname{Pr}\left(U_{\bar{a}} \text { is a sub-witness }\right)+\operatorname{Pr}\left(U_{\bar{a}} \text { is a complaisant flat }\right),
$$

where

$$
\begin{aligned}
\operatorname{Pr}\left(U_{\bar{a}} \text { is a sub-witness }\right) & :=2^{e-n} \cdot \prod_{i=1}^{s} \frac{2^{e}-2^{i-1}}{2^{n}} \\
\operatorname{Pr}\left(U_{\bar{a}} \text { is a complaisant flat }\right) & :=2^{-2^{s}+1} \cdot \frac{2^{n-e} N S(n, s)-N F(e, s)}{N S(n, s)} .
\end{aligned}
$$

In the above formula, $e$ is the dimension of the witness. The formulas for $N S(\cdot, \cdot)$ and $N F(\cdot, \cdot)$ are given in Lemma 7. 
Proof: We first determine the probability that the flat $U_{\bar{a}}$ is a sub-witness. This probability is justified with an inductive argument on the dimension of the sub-witness: for one point (i.e., a flat of dimension 0), the probability of being a sub-witness is $\frac{\mathbf{2}^{\mathbf{e}}}{\mathbf{2}^{\mathbf{n}}}$. Here, the witness has $2^{\boldsymbol{e}}$ points. This probability is also true for extending the subwitness from dimension $(i-1)$ to dimension $i$ (we have $1 \leq i \leq s$ ). In addition, we have to consider the case $\bar{p}_{i} \in \bar{p}_{0}+\left\langle\bar{p}_{1}, \ldots, \bar{p}_{i-1}\right\rangle$, i.e., the new point $\bar{p}_{i}$ lies in the sub-witness of dimension $(i-1)$ generated by the points $\bar{p}_{0}, \ldots, \bar{p}_{i-1}$.

The probability that $U_{\bar{a}}$ is a complaisant flat is equal to the probability that the function is constant on $U_{\bar{a}}$ times the number of flats which are parallel with a witness but not part of a witness. This is exactly expressed in the formula.

$>$ From the previous theorem and the implicit evaluation strategy as described in Sect. 3.2, we can deduce the following corollary.

COROLLARY 10 For a given start dimension $\boldsymbol{s}$ and an end dimension $\boldsymbol{e}$, we need at most

$$
\operatorname{Rep}(n, s, e, c)=\frac{c}{\operatorname{PF}(n, s, e)} \cdot \frac{1}{\operatorname{Pr}(\text { only 1 flat }) \# \text { flats }}
$$

repetitions to achieve a confidence of $2^{-c}$ that the function $f$ is not e-normal.

Table 2 shows some numerical values of $r$ in $\log _{2}$. In this and all following tables, we concentrate on even choices for $n$ and fix $e=\frac{n}{2}$ as these cases are particularly relevant in cryptography.

Table 2. Number of repetitions (in $\log _{2}$ ) for different values of $n$ and $s$

\begin{tabular}{|c||c|c|c|c|c|c|c|}
\hline$s \backslash n$ & 8 & 10 & 12 & 14 & 16 & 18 & 20 \\
\hline \hline 2 & 15.49 & 18.35 & 21.28 & 24.25 & 27.23 & 30.22 & 33.22 \\
\hline 3 & 18.68 & 22.31 & 26.14 & 30.06 & 34.02 & 38.00 & 41.99 \\
\hline 4 & & 26.11 & 30.72 & 35.54 & 40.45 & 45.40 & 50.38 \\
\hline
\end{tabular}

\section{Complexity of the main loop.}

Obviously, picking $(2 s+1)$ random points and checking if the function is constant for a given flat, will be the most expensive operations. Therefore, we start with a lemma on the average complexity for checking that a function is constant on a given set of points.

LEMMA 11 For a given random function $f: \mathbb{F}_{2}^{n} \rightarrow \mathbb{F}_{2}$ and a given set of points $P \subseteq \mathbb{F}_{2}^{n}$, the algorithm from Fig. 6 needs on average 3 evaluations of $f$ to check if this function is constant when restricted to vectors in the set $P$.

Proof: The average number of evaluations depends on the number of points $p:=|P|$ of this algorithm; it is given by

$$
E v(p):=\sum_{i=1}^{p-1} \frac{1}{2^{i}}(i+1)+\frac{1}{2^{p-1}} p=3-\frac{1}{2^{p-2}} .
$$


Figure 6. Algorithm to determine if a function is constant on a set of points

Input: $\quad$ function $f$, a set $P$ with $p:=|P|$ points

Output: 1 if $f$ is constant on $P$ and 0 otherwise

Let $\bar{q}_{1} \in P, c \leftarrow f\left(\bar{q}_{1}\right)$

for $\bar{q} \in P \backslash\left\{\bar{q}_{1}\right\}$ do

if $f(\bar{q}) \neq c$ then OUTPUT 0

OUTPUT 1

To justify this formula, we observe that we need to evaluate $f$ at least once to obtain the constant $\boldsymbol{c}$. As the function is a random function by definition, we have a probability of $\frac{1}{2}$ to obtain a different constant for every further evaluation, i.e., to terminate this algorithm. After checking a total of $\boldsymbol{p}$ points, the algorithm terminates. For this last check, we still have a probability of $\frac{1}{2}$ to output 0 . However, the workload of outputting 0 or 1 is exactly the same, namely $\boldsymbol{p}$ evaluations.

As a consequence, the complexity of the main loop so far depends on the costs of picking the $(2 s+1)$ random points, evaluating the function $f$ on the corresponding flat with probability $\operatorname{Pr}($ Only one flat) and some other negligible operations whose complexity we set to one, i.e., $(2 s+1+3 \operatorname{Pr}($ Only one fiat $)+1) r$, where $r$ represents the number of repetitions. We obtain the following values $\left(\log _{2}\right)$ if we evaluate the above formula numerically (cf Table 3).

Table 3. Numerical results for the time-complexity (in $\log _{2}$ ) of the main loop

\begin{tabular}{|r||c|c|c|c|}
\hline \multicolumn{1}{|c||}{$n$} & $s=2$ & $s=3$ & $s=4$ & $s=5$ \\
\hline \hline 8 & 18.47 & 21.95 & & \\
\hline 10 & 21.33 & 25.58 & 29.63 & \\
\hline 12 & 24.26 & 29.41 & 34.24 & 39.12 \\
\hline 14 & 27.23 & 33.33 & 39.06 & 44.72 \\
\hline 16 & 30.21 & 37.29 & 43.97 & 50.53 \\
\hline 18 & 33.20 & 41.27 & 48.93 & 56.44 \\
\hline 20 & 36.20 & 45.26 & 53.90 & 62.40 \\
\hline
\end{tabular}

\section{Complexity of the SearchForParallelFlats-method.}

From a computational point of view, the for-loop is very expensive, as we have to check $2^{n-s}-1$ parallel flats every time. However, each flat costs only 3 operations on average (cfLemma 11). In addition, we only need this for-loop in $2^{-2^{s}+1}$ of all cases as this is the probability that the function is constant on the corresponding flat. The other steps in the method are negligible in comparison to the for-loop. We therefore identify their average workload as 1 . Consequently, the complexity can be approximated by $\left(1+3 \cdot\left(2^{-2^{s}+1} \operatorname{Pr}\right.\right.$ (only one flat $\left.\left.)\right) 2^{n-2^{s}-s+1}\right) r$ for the Search For Parallel Flatsmethod, where $\boldsymbol{r}$ denotes the number of repetitions. Numerical values for the timecomplexity (in $\log _{2}$ ) of the SearchForParallelFlats-method are presented in Table 4. 
Table 4. Numerical results for the time-complexity (in $\log _{2}$ ) of the SearchForParallelFlatsmethod

\begin{tabular}{|r||c|c|c|c|}
\hline$n$ & $s=2$ & $s=3$ & $s=4$ & $s=5$ \\
\hline \hline 8 & 19.50 & 19.18 & & \\
\hline 10 & 24.28 & 23.71 & 26.11 & \\
\hline 12 & 29.20 & 29.06 & 30.73 & 35.38 \\
\hline 14 & 34.16 & 34.83 & 35.60 & 40.98 \\
\hline 16 & 39.14 & 40.75 & 40.69 & 46.79 \\
\hline 18 & 44.13 & 46.72 & 46.20 & 52.70 \\
\hline 20 & 49.13 & 52.71 & 52.37 & 58.66 \\
\hline
\end{tabular}

\section{Complexity of the Combine-procedure.}

The complexity analysis of the combine-procedure is a little more tricky. In particular, we have to deal with the problem that its complexity depends quadratically on the number of parallel flats we find, i.e., the number $\left|P\left(U_{\bar{a}}\right)\right|$ for a given flat $U_{\bar{a}}$. Therefore, we cannot simply take the average number of flats for this analysis as the result does not reflect the real time complexity of this algorithm. In addition, we have to deal with the branching condition (cf Sect. 3.4).

As we did not expect to find a closed formula for the time complexity of the combine-procedure, we used MAG to compute it numerically. As all computations are done with rational numbers, there are no rounding errors in MAGMA. In particular, we computed the probability for the different numbers of parallel flats we obtain in the searchForParallelFlats-method. We only took numbers $\geq \mathbf{2}^{\boldsymbol{e} \boldsymbol{- s}}$ into account (cf Thm. 6) and neglected levels of recursion which appear with too small probability $\left(<2^{-40}\right)$, due to the branching condition. In addition, we truncated the sum at points which did not contribute to the overall workload anymore (expected workload smaller than 1). We present the corresponding values $\left(\log _{2}\right)$ for different choices of $n$ and $s$ in Table 5 .

Table 5. Numerical results for the time-complexity (in $\log _{2}$ ) of the Combine-method

\begin{tabular}{|r||c|c|c|c|}
\hline$n$ & $s=2$ & $s=3$ & $s=4$ & $s=5$ \\
\hline \hline 8 & 24.17 & 15.97 & & \\
\hline 10 & 31.15 & 22.87 & $\approx 0$ & \\
\hline 12 & 38.03 & 15.76 & $\approx 0$ & $\approx 0$ \\
\hline 14 & 44.97 & 23.68 & $\approx 0$ & $\approx 0$ \\
\hline 16 & 51.93 & 35.02 & $\approx 0$ & $\approx 0$ \\
\hline 18 & & 43.34 & $\approx 0$ & $\approx 0$ \\
\hline 20 & & 51.33 & $\approx 0$ & $\approx 0$ \\
\hline
\end{tabular}

These computations were matched by our empirical results. In particular, the branching condition proved to be very powerful for $s \geq 3$ and $n \geq 12$ (note difference between $n=10$ and $n=12$ for $s=3$ ). In these cases, we never needed a recursive call of the combine-method for non-normal functions. In addition, the probability for a function to be constant on a given flat decreases exponentially with 
increasing dimension of the flat. Therefore, we expect to find less than $2^{\boldsymbol{e}-\boldsymbol{s}}$ flats for $s \geq \mathbf{4}$ and $n \leq 20$ which means that the combine-method is never invoked in these cases (fields with $\approx 0$ in the above table).

All in all, it is necessary to chose the starting dimension $\boldsymbol{s}$ correctly, i.e., high enough such that the combine-method is still efficient and low enough such that SearchForParallelFlats and the main loop do not need too much time. For dimension $n \geq 10$, the choice $s=3$ turns out to be optimal (cf Fig. 7 for the case $n=16$ ).

Figure 7. Time-complexity for the main loop (๑), SearchForParallelFlats (*), and the combine-method $(x)$ for dimension $n=16$ and varying $s$

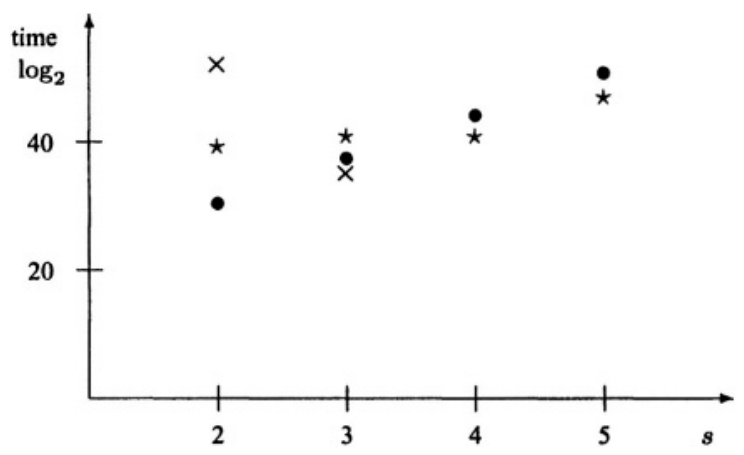

\section{Asymptotic Analysis.}

Here we sketch the asymptotical analysis of the above algorithm: we begin with the observation that for large $\boldsymbol{n}$ and subsequently large $\boldsymbol{s}$, the running time will only depend on the number of repetitions necessary. We justify this reasoning as follows: as we saw for the combine-method, we have a very powerful branching condition, i.e., asymptotically, this part will not contribute to the overall complexity. The same is true for the search of parallel flats: we have a complexity of $O\left(2^{\left(-2^{\circ}+1\right)(n-s)}\right)$ here, i.e., negligible for $\boldsymbol{n} \rightarrow \infty$. In addition, we cannot use the implicit evaluation strategy anymore in the asymptotic case, as we obtain a rather small probability for having exactly one flat $s \rightarrow \infty$. Therefore, we drop the corresponding term in our asymptotic analysis. For our analysis, we chose $s=\frac{1}{4} n$ and $\boldsymbol{e}=\frac{1}{2} n$ and obtain the following asymptotically upper bound on the number of repetitions and thus the running time of the algorithm:

$$
\operatorname{Rep}\left(n, \frac{1}{4} n, \frac{1}{2} n, c\right)=O\left(c .2^{t^{n^{2}+\frac{3}{4} n}}\right),
$$

where $\boldsymbol{c}$ is the target confidence level. To obtain this upper bound, we observe that the probability to have a complaisant flat is asymptotically very small. In addition, we notice that for large $n$ the factor $2^{e-n+s(e-1-n)}$ is a tight lower bound on the probability $P F(n, s, e)$. Using Theorem 9 and Corollary 10 yields the result. 


\subsection{Comparison with the Algorithm from Daum et al.}

In Fig. 8 and Table 6 we compare the time complexities of our algorithm with that of DDL03, for computing the normality of a function in dimension $n$. We are not aware of an asymptotical analysis of the algorithm from DDL03.

Figure 8. Time-complexity (in $\log _{2}$ ) of this paper ( $\star$ ) and from DDL03 (•)

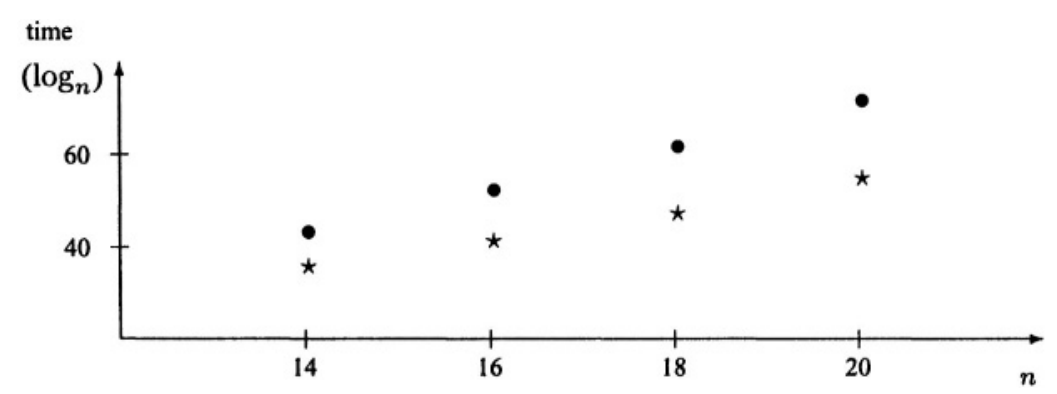

The time complexity of algorithm of DDL03, is computed using the formulas given there. According to these results, we expect that it is outperformed by our algorithm for increasing dimension $n$.

Table 6. Comparison of the time-complexity (in $\log _{2}$ )

\begin{tabular}{|c|c||c|c|}
\hline$n$ & $s$ & Daum et al. & Our alg. \\
\hline 14 & 2 & $\mathbf{4 2 . 5 8}$ & 44.97 \\
& 3 & $\approx 46$ & 35.27 \\
& 4 & $\approx 52$ & 39.18 \\
\hline 16 & 2 & $\mathbf{5 1 . 5 8}$ & 51.93 \\
& 3 & $\approx 54$ & 40.88 \\
& 4 & $\approx 62$ & 44.11 \\
\hline
\end{tabular}

\begin{tabular}{|c|c||c|c|}
\hline$n$ & $s$ & Daum et al. & Our alg. \\
\hline 18 & 2 & 61.17 & $>50$ \\
& 3 & 61.01 & 46.72 \\
& 4 & $>61$ & 49.13 \\
\hline 20 & 2 & 71.09 & $>55$ \\
& 3 & 71.04 & $>55$ \\
& 4 & $>71$ & 54.33 \\
\hline
\end{tabular}

\subsection{Empirical Results}

We have implemented our algorithm in a programme with 14,000 lines of $\mathrm{C}++$ code. Checking random functions on an AMD Athlon XP 2000+, we obtained the following results for $e=\frac{n}{2}$ (normality) and $s=3$ :

\begin{tabular}{|r|c|c|c|c|}
\hline$n$ & 10 & 12 & 14 & 16 \\
\hline time [min] & 0.248 & 1.21 & 42.6 & 2880 \\
\hline
\end{tabular}

As we see in this table, the running time gets quickly out of hand. According to DDL03, their programme needs approximately $50 \mathrm{~h}$ on a Pentium IV $1.5 \mathrm{GHz}$ 
for the case $n=14$. Our algorithm needs approximately $43 \min$ for $n=14$ and approximately $2 \mathrm{~d}$ for $\boldsymbol{n}=\mathbf{1 6}$. Using the complexity analysis of DDL03, we expect a running time of more than a year for their algorithm to handle functions of dimension $n=16$. We also estimated (empirically) the running time for the cases $n=18,20$ and obtain 2.5 years and 130 years, respectively.

For our $\mathrm{C}++$ implementation, we have included several improvements:

Combinatorial Gray codes. In order to compute vectors more efficiently for a given basis, we used combinatorial Gray codes (Sav97) and computed all intermediate values in a Gray code like fashion. This way, we only needed one computation on average rather than $\frac{n}{2}$ when computing elements of the vector space $\langle\bar{U}\rangle$.

Optimised Pseudo-Random Number Generator. As the programme spends approx. $60 \%$ of its time computing random numbers, we concluded that it could benefit from a fast way of generating pseudo-random numbers. However, due to the high number of repetitions, we still need a long period for the pseudo-random number generator. To meet both aims, we used a pseudo-random number generator from Rho which combines a multiply with carry generator and a simple multiplicative generator. It achieves a period of more than $\mathbf{2}^{\mathbf{6 0}}$, has good statistical properties, and is also very fast according to our measurements. For the future, tests with the cryptographically secure pseudo-random number generator using Shamir's T-functions class (KS04) are planned.

Function storage. For the Boolean function to be checked, we can use several ways of storing it: bit-wise, byte-wise or in processor-words ( $32 \mathrm{bit}$ ). To make the best use of the internal cache of the processor, a bit-wise storage turned out to have the best performance for dimensions $\boldsymbol{n} \geq \mathbf{1 2}$. For dimensions $n \leq 10$, an word-wise storage was clearly better as we do not have the overhead of retrieving single bits from a word.

\section{Conclusions}

In this paper, we present a fast asymmetric Monte Carlo algorithm to determine the normality of Boolean functions. It uses the fact that a function which is constant on a flat of a certain dimension is also constant on all sub-flats of lower dimension. In addition, we evaluate "parallel" flats using the implicit evaluation strategy (cf Sect. 3.2). Starting with flats of dimension $\boldsymbol{s}$ and combining them until a flat of dimension $\boldsymbol{e}$ is obtained, we achieve a far lower time-complexity than with exhaustive search on flats of dimension $\boldsymbol{e}$.

In particular, this algorithm is far faster than the previously known algorithm (43 min in comparison to $50 \mathrm{~h}$ ) for dimension 14 (cf4.2). Moreover, it is the first time that the important case $\boldsymbol{n}=16$ can be computed on non-specialised hardware in 2 days (previously: more than a year). Using the fact that our algorithm can be parallelised easily, this figure can even be improved and we can even handle the case $\boldsymbol{n}=\mathbf{1 8}$ (16 computers in 8 weeks). For scientific purposes and at present, $\boldsymbol{n}=\mathbf{2 0}$ seems to be out of reach as it would take 128 computers about 1 year. 


\section{Acknowledgments}

We want to thank the authors of DDL03, for helpful remarks and sending us both an early and an extended version of their work.

The authors were partially supported by Concerted Research Action GOA-MEFISTO666 of the Flemish Government and An Braeken is research assistant of the Fund for Scientific research - Flanders (Belgium).

\section{References}

Canteaut, Anne; Daum, Magnus; Dobbertin, Hans; and Leander, Gregor (2003). Normal and non-normal bent functions. In WCC03. 19 pages.

Carlet, Claude (2001). On the complexity of cryptographic Boolean functions. In $6^{\text {th }}$ Conference on Finite Fields and Applications, $21^{\text {th }}-25^{\text {th }}$ May, pages 53-69. Gary L. Mullen, Henning Stichtenoth, and Horacio Tapia-Recillas, editors, Springer.

Daum, Magnus; Dobbertin, Hans; and Leander, Gregor (2003). An algorithm for checking normality of Boolean functions. In WCC03. 14 pages.

Dobbertin, Hans (1994). Construction of bent functions and balanced Boolean functions with high nonlinearity. In Fast Software Encryption - FSE 1994, volume 1008 of Lecture Notes in Computer Science, pages 61-74. Bart Preneel, editor, Springer.

Dubuc, Sylvie (2001). Etude des propriétés de dégénérescene et de normalité des fonctions booléennes et construction des fonctions q-aires parfaitement non-linéaires. $\mathrm{PhD}$ thesis, Université de Caen.

Kipnis, Aviad and Shamir, Adi (2004). New cryptographic primitives based on multiword Tfunctions. In Fast Software Encryption - FSE 2004. Bimal Roy and Willi Meier, editors. pre-proceedings, 14 pages.

Landau, David and Binder, Kurt (2000). A Guide to Monte Carlo Simulations in Statistical Physics. Cambridge University Press. ISBN 0-521-65314-2.

MacWilliams, F.J. and Sloane, N.J.A. (1991). The Theory of Error-Correcting Codes. Elsevier Science Publisher. ISBN 0-444-85193-3.

MAGMA. The MAGMA Computational Algebra System for Algebra, Number Theory and Geometry. Computational Algebra Group, University of Sydney. http: //magma.maths.usyd.edu.au/magma/ .

Rhoads, Glenn. Random number generator in $\mathrm{C}$. http: //remus. rutgers. edu/ rhoads/Code/rands.c.

Savage, Carla (1997). A survey of combinatorical Gray codes. SIAM Review, $39(4): 605--629$.

http: / /www.csc.ncsu.edu/faculty/savage/AVAILABLE_FOR_MAILING/survey.ps .

WCC (2003). Workshop on Coding and Cryptography 2003.

Daniel Augot, Pascal Charpin, and Grigory Kabatianski, editors, l'Ecole Suprieure et d'Application des Transmissions.

ISBN 2-7261-1205-6. 